undoubtedly high state of civilization on the part of the builders.

The Valley of Mexico is a basin of about seventy miles in length and forty-five miles in width. It extends from Pachuca on the northeast to the mountains south of Lake Chalco, and from the Sierra Nevada range on the east to the Sierra de los Cruces range on the west. The valley has an area of 1,750 square miles, or is about one-fourth greater than the State of Rhode Island. In the northern half are three small lakes and scores of small villages, but no large towns except Pachuca; in the southern half are three large lakes, many large villages and the City of Mexico. The lowest portion of the basin is Lake Texcoco, which in its normal condition is only six feet below the level of the city. The bottom of the valley has an elevation of 7,400 feet, while the highest point on the sides is about 17,777 feet above the level of the sea. We enter the northern half of the valley through the Cut of Nochistongo, and the southern half through an opening on the western side of Sierra Guadalupe.

In this valley we are in the earliest home of man on this continent. The Chichimecs settled there in A. D. 635, a hundred years after the Toltecs came, and these were succeeded by the Aztecs in A. D. 890 . Their name for the region was Anahuac, signifying "the place of waters."

Beside Texcoco, there are five other lakes in the valley. Lake Zumpango is the highest in the valley and is east of the cut. The River Cuatitlan emptied into the Zumpango, but the cut was made so as to turn the river's course into the Tula River. The new canal carries the water of the valley into the Ravine of Tequixquiac before entering the Tula River.

Zumpango is thirty miles north of Mexico City; Xaltocan and Zumpango are at an elevation of twentyfive feet above the city. San Cristobal is a small lake immediately north of Texcoco, twelve miles N.N.E. of Mexico and is ten miles long. Chalco and Xochimilco are the southernmost, whose levels are ten feet above Texcoco. The largest and nearest is but six feet below the pavement of the city at ordinary stages of the water. Texcoco is a salt-water lake two and onequarter miles east of the City of Mexico, with which it is connected by a canal; its greatest length from north to south is about fifteen miles, its greatest width nine miles. Its depth nowhere exceeds ten feet, and in some parts is not more than two or three feet, while its shores are swampy. The lake is said to have been much more extensive than at present and to have included several islands, on which the Mexico of the Incas was built. Lake Texcoco is without any natural outlet.

I should say that there were presented in the Section on Anatomy, including Physiology, two papers of unusual interest. One of these was on "Hematology of High Altitudes," by Dr. Galdino Casillas, Guadalajara, Mexico. He said that "the increase of red blood corpuscles in the system in elevated climates is real and not fictitious," that is, that the condition of the system in elevated regions is not a "physiologic concentration of blood discs from a loss of water."

The other of these papers was on "Anatomic and Physiologic Modifications Due to the Elevation," by Dr. Daniel Vergara Lope together with Prof. Alfonso L. Herrera of Mexico. They referred to the "increase of the biachromeal distance, of the length of the ster- num, of the clavicular widening of the thorax and the increase of the pulmonary capacity." Those authors referred to the relation of the thorax to the height, and to several other vital conditions that obtain in the organism of those who dwell in high altitudes. The summary was that man can become completely acclimated to elevated centers. His humors are con. centrated, his respiration and circulation are accelerated and the decrease of oxygen is compensated for by the increased activity of the hematosis. These facts, they said, ought to be borne in mind by the physician who is established in high altitudes. If these conclusions are correct, may not the varying degrees of the stature of individuals be dependent in large measure upon the degrees of altitude to which the progenitors during a long course of ages have been subjected?

\section{APPENDICITIS, WITH REPORT OF AN INTERESTING CASE.}

Read before the Section on Abdominal Surgery, Obstetrics and Gynecology, Second Pan-American Medical Congress, Mexico City, Mexico, Nov. $18,1896$.

BY HENRY L. E. JOHNSON, M.D.

A Vice-President of the Second Pan-American Medical Congress; late President of the Clinico-Pathological Society; late Vice-President of the Washington Obstetrical and Gynecological society: Professor of Gybecology in the Medical Department of the Columbian University: Professor of Gynecology in the Post-Graduate School of Medicine of the District of Columbia; Director of the Cynecologic Clinic in the Central Dispensary and Emergency Hospi-
tal; Consultant to the Woman's Clinic, etc. WASHINGTON, D. C.

I have the pleasure, Mr. President and fellow members, of presenting for your consideration and criticism a few remarks and observations on appendicitis, the disease which is attracting so much attention at the present time, and which is possibly not thoroughly understood by the medical profession of the day. The disease is one of frequent occurrence, and its mortality is in direct ratio to the accuracy of diagnosis and treatment.

Nomenclature.-The disease is and has been variously known as iliac phlegmon, typhlitis, perityphlitis, paratyphlitis, cecitis, pericecitis, paracecitis, periappendicitis, ecphyaditis and appendicitis.

History.-M. Mastivier, in Journal of Medicine, Surgery and Pharmacy, Paris, May, 1759, reports the case of a man aged 45, with a large tumor in the right side near the umbilicus, who presented himself for treatment at the Hospital of St. Andrew of Bordeaux in the year 1757. Operation: evacuation of about a pint of offensive pus; postmortem showed a gangrenous cecum and appendix, the latter containing a large corroded and encrusted pin.

Early in the present century "Phlegmons in the Right Iliac Fossa" were extensively written about, without, however, attributing the same to the appendix. In 1831 Farrall published a paper on "Phlegmonous Tumor in the Right Iliac Region," in which the cecum is set down as the cause of the disease. In 1833 Dupuytren gave expression to the same views. In 1834 Copeland recognized the appendix as a possible factor, but quite secondary to the cecum. In 1837 the ulceration of the appendix from foreign bodies is spoken of as a matter of little consequence so long as confined to the mucous membrane, but causing abscesses when the peritoneum is involved. Hancock operated on one case in 1848. In 1867 Willard Parker of New York favors an early operation. From this time on, the theory of the cecum diminished and that 
of the appendix increased. In 1880 Woirth of Copenhagen expressed his disbelief in the cecal theory. In 1883 Dr. F. F. Noyes reports 100 cases, of which 90 per cent. were operated upon in America. In 1886, Fitz of Boston put on record 209 cases.

Etiolofy.-Appendicitis is more frequent in males than in females. The disease is most common between the ages of 10 and 20 . Fifteen per cent. of all cases occur in people under 15 years of age. Among the anatomic causes which predispose to inflammation of the appendix, the blood supply forms an important part, there being only one artery supplying the organ, and that forming no anastomosis. The richness of the lymphatic tissue which is found in the appendix plays an important part. Finally, it is claimed that its position favors inflammation.

Predisposing causes.-Intestinal catarrh and indigestion; distension of bowel with chronic constipation.

Exciting causes.-The usual causes assigned are the lodging of foreign bodies, fecal concretions, microorganisms and acute intestinal indigestion from imprudence in diet.

Varieties and pathology.-Various forms of the disease are recognized by different authors, the principal ones are catarrhal, ulcerative, infective and recurrent appendicitis, and the following seems to be the usually recognized pathology:

Catarrhal.- The exact frequency of this form, when mild, can not be determined. It probably produces no symptoms and may subside without leaving a trace. Chronic catarrh is, however, frequently found on postmortem examinations and is easily recognized, as it gives a thickening to the wall. The actual changes are those of mucous membranes elsewhere. There occurs a rapid shedding of the epithelium with detachment and extrusion of the epithelial lining of the crypts of Lieberkühn. The base of the mucous membrane is infiltrated by leucocytes, and the crypts are obliterated. The lumen is found to contain an abundance of leucocytes, granular débris, mucus and casts of the interior of the crypts, and the whole is sometimes molded into a definite central mass by the muscular movements of the tube. Perfect recovery by a new growth of epithelium is at first possible, but the shedding may be so complete and over so large an area that the denuded surface can not be covered anew. The inner part of the mucous surface now comes to consist of grarulation tissue. This condition happily acts often as a preventive of further attacks, as by pressure of the surrounding parts the two surfaces grow together, with obliteration of the lumen of the tube, or again fibrous tissue may obliterate the cavity, and a natural cure results. The process may be confined to only a part of the tube, in which case there will be a stricture, usually near the cecal ends. The remaining portion sometimes is largest, forming a cyst. In many cases the condition becomes chronic with great thickening of the wall, as much as one-eighth to one-tenth of an inch in some instances, rendering the appendix firm and rigid.

Ulcerative.-This form is not preceded by a general catarrh. The ulceration is due to fecal concretions or foreign bodies. Feces press on the wall and injure the epithelium, which is ulcerated and finally destroyed. The mucous membrane, submucous tissue and finally the muscular coats disappear. In twentyseven fatal cases (St. Thomas' Hospital) fecal concretions were found in eleven. Fitz (Boston), in
152 cases found them in 47 per cent. Mathews in 63 per cent. of 169 cases.

Infective.-This form of appendicitis may result from either one of the preceding, or may have an independent origin. It seems to be associated with bacterial invasion of the tissues of appendix wall, and in all cases leads to a virulent peritonitis. The bacillus usually most active is the coli communis.

Recurrent appendicitis.-This form is prominently known but quite recently. Treves has reported a case in which there were fourteen attacks, and McBurney reports one in which twelve attacks occurred within as many months. Krafft states that of 106 cases which he studied, 23 per cent. had been previously affected. The writer reports herein one case in which five relapses occurred within twelve months. Operation was successfully performed during the fifth attack.

The writer believes that all cases originate in a catarrh or inflammation resulting from torsion, or volvulus of the tube or from fecal or other concretions, all producing greater or less ulceration, with sometimes perforation and subsequent bacillus infection of the appendix and adjacent tissues. The rapidity and quantity of pus formation is familiar to all surgeons, two or three pints having been known to form in some cases by the fifth day of the disease. The writer believes the process in the appendix follows a similar course with the Fallopian tubes, viz., inflammation with contractions, puckerings, obstruction and fluid accumulation with in some cases resolution, and in others the formation of an infected pus cavity, which bursts or perforates and on the one hand results in general peritoneal infection, or by adhesive limitation, forms a circumscribed abscess or abscesses. Sometimes the appendix is found diseased and even gangrenous and without adhesions. In other instances the appendix is almost disorganized and is surrounded by pus and quite free from adhesions. The writer has seen such a case recently in consultation in which the appendix was removed and the patient recovered. The adhesions and torsions of the appendix so often observed in abdominal sections in gynecologic practice, may sooner or later be a factor in the causation of this disease.

Previous attacks of appendicitis which appear to have undergone resolution, predispose to subsequent attacks of greater or less gravity. Perforation with septic infection of the peritoneum and death may result very early in the attack. Matterstock found perforation in 132 out of 146 cases, while Fenwick found it to exist in 113 out of 130 cases. Accurate pathology will be obtained only by early and frequent operations and careful laboratory study of specimens. Symptomatology.-The symptoms are usually severe and very pronounced. Pain, sudden and severe, is the first symptom noted. It is not always in the right iliac region, though at the end of twenty-four hours it usually becomes most prominent there. There is usually headache, sometimes a chill. Vomiting may or may not be an urgent symptom. The initial tem. perature usually ranges from 100 to 104 degrees, with a tendency to fall within two or three days. Sometimes the temperature is low and not in proportion to the other symptoms. The pulse is usually rapid, sometimes intermittent. Tenderness on pressure in right iliac region, with marked tension or rigidity of abdominal muscles over the right iliac region is almost. pathognomonic. In $1889 \mathrm{McBurney}$ first drew atten. tion to a spot at which on deep pressure pain was 
always elicited. This spot is situated at from one and one-half to two inches from the anterior superior spine of the right ilium along a line drawn from that process to the umbilicus. Constipation is usually marked at first, but may be preceded by two or three loose movements. Abdominal distention is usually marked for the first day or two. Increasing disten. tion is a bad symptom. The pain is later increased upon motion or coughing, and there is a tendency to flexion of the right thigh or drawing the leg up to the body. The patient is most comfortable when lying upon the back. There is usually a tumor in the right iliac region of varying size, which is detected from the first to the third day. It consists of the appendix, intestines, omentum and exudate, and usually increases in size as the disease progresses. Exaggeration or increasing intensity of pain, accompanied by symptoms of shock, with the disappearance of tumor, followed by chill, fever, tympanites and vomiting, indicate usually rupture in to the peritoneal cavity. These symptoms to a less degree, however, may indicate the formation of pus without perforation or perforation into the bowel. Prostration may or may not be a prominent symptom. The condition of the urine is usually negative, but sometimes contains albumin. There is usually marked loss of appetite with increased thirst. There are, sometimes, prodromal symptoms manifested for a few days, consisting of abdominal uneasiness and soreness with some distention and flatulence. These may often be discovered to have existed without attracting the patient's attention particularly.

Diagnosis.-The diagnosis is based upon the history of the case, the physical signs and the symptoms above mentioned.

Differentiation. - Appendicitis must be differentiated from renal and hepatic colic, acute indigestion, cholera morbus, strangulated inguinal hernia, torsion and volvulus and obstruction of bowels, and the various acute pelvic diseases in women.

Termination and Prognosis. - Fitz says that 26 per cent. and Stimson that 25 per cent. of all cases of appendicitis prove fatal. According to Fitz, 60 out of 176 cases of perforated appendicitis died during the first five days, 56 during the first four days, 28 during the first three days, and 8 during the second day, though a large number of cases of simple appendi. citis are said to terminate favorably by resolution. Bull, in 1894, placed the mortality at 5 to 6 per cent., although he had collected 450 cases with only 8 deaths. Deaver collected 200 cases with only 2 deaths. Robert T. Morris records the mortality as 15 per cent. under medical treatment, with an addition of 10 per cent. from numerous chronic complications resulting from previous acute attacks. The prognosis is consequently grave and will depend upon early diagnosis and prompt modern surgical interference.

Treatment.-The majority of writers concur in the opinion that there is no reliable medicinal treatment for appendicitis. Osler says ("Principles of Medicine," 1895): "There is no medicinal treatment for appendicitis. Operation is indicated for acute inflammatory trouble in cecal region whether a tumor is present or not, and when by the third day the features of the case point to a progressive lesion." The case once accurately diagnosticated, most writers agree that the operation should be done at once. In cases of recurring appendicitis the operation is performed in the interval, or as soon as the symptoms recur. When the appendix can be found and its removal does not entail the breaking up of adhesions, thereby opening the peritoneal cavity, it is removed in the usual way. When not removed, the case is treated as an abscess cavity, being packed and drained. In this treatment there seems to be a consensus of surgical opinion. Thus Dr. J. D. S. Davis, of Birmingham, Ala., in Medicine for February, 1896, says: "To make an extensive search for the appendix is liable to break up adhesions and allow the escape of septic pus into the general cavity. The records of operation upon appendical abscess show that the great majority of cases are cured after evacuation and complete drainage."

Dr. J. B. Deaver (Surgeon to the German Hospital, Philadelphia): "The best results are obtained when the operation is performed at the earliest opportunity."

According to Ribbert 16 out of 400 cases recover without operation. Deaver recommends the removal of the organ in all cases if the operator be a skilful one! Otherwise he simply evacuates the pus and drains the cavity.

"Murray believes the mortality to be 1 per cent. When he finds a walled-in abscess in the inguinal region, the abscess cavity is cleansed with hydrogen peroxid and flushed with hot saline solution, whereupon search may be made for the appendix and multiple abscesses in the vicinity: without the danger of infecting the abdominal cavity when the adhesions are broken down."

G. M. Gray (Kansas Medical Journal of December, 1895) "removes the appendix if the operation is done early; if late he opens the cavity and drains. The question of removing the appendix in these cases to be determined by the operator for each individual case."

Dr. J. William White of Philadelphia says: "In a circumscribed abscess it is bad surgery to insist on finding the appendix; should be content with treating as an abscess." He (White) has left the appendix in thirty-seven cases, all of which are living. Dr. White's treatment and teaching is thus supported.

Richardson reports forty cases with the appendix left; perfect recovery in all.

McBurney says, "the appendix is frequently destroyed before the operation." If in such cases indefinitely prolonged search were to be made for the appendix fatal results would be inevitable.

Dr. Bull says: "The plan of always looking for the appendix is fraught with the risk of infecting a healthy peritoneum, and that the mortality from surgical treatment is about 2 per cent. in cases operated upon at a time when the infection is limited to the confines of the appendix."

Dr. Senn states that " persistent search is hazardous." Halstead says the same. Cabot of Boston reports thirty-two operations on thirty-one patients in the interval of the attacks, with no deaths. Hunter McGuire reports seventeen operations with one death from chronic appendicitis.

The writer holds that these cases are not prejudiced unfavorably by early operation, nor is the danger to thc patient increased, hence, all cases should be operated upon at once. Even in cases of mistaken diagnosis, operation under thorough aseptic precautions will be attended with no more danger than an exploratory section, and notwithstanding the fact that the appendix is found to be normal it should be removed, 
thus preventing a possible future attack of appendicitis. When the appendix is found to be diseased, it should be removed and treated by immediate closure of the abdomen by suture, preferably silkworm gut where pus is not present, and by drainage by gauze packing without drainage tube, where pus is present. In the case of a large abscess or multiple abscesses the correct treatment is drainage, after thorough flushing with saline solution, and no hazardous attempt should be made to secure an appendix which has in all probability sloughed away. Multiple abscesses should be carefully converted into one large cavity and so treated. Should the appendix or a portion of it remain and produce subsequent trouble, a second operation should be performed at once.

In cases where the abdomen is opened for other causes the appendix should always be removed as a prophylactic measure, and the danger from the original operation is not thereby increased, the "pus cases" found in women not excepted. Every attention should be given to secure absolute asepsis both before, during and after operation. The subsequent treatment is that recognized in abdominal surgery. In the case of abscesses the greatest judgment should be exercised in selecting the point for incision, thereby avoiding the danger of opening too near or perforating the exudate boundary of the abscess, thus infecting the general peritoneum.

HISTORY OF AN INTERESTING CASE OF RECURRENT APPENDICITIS, RECOVERY AFTER OPERATION.

Mr. - age 37. Previously in excellent health. Had typhoid fever about thirteen years ago and was quite ill, with marked abdominal symptoms, but recovered without complications. Had his first attack of appendicitis on Aug. 2, 1895. The attack was ushered in very much like an attack of colic from indigestion, and followed by acute pain over entire abdo. men, with nausea, vomiting and purging. By the next day the general symptoms had more or less subsided and the disorder was clearly defined in the right iliac region. The illness lasted one week and subsided under rest, diet, opiates and enemas. The diagnosis of catarrhal appendicitis was made.

A second attack occurred on Sept. 1, 1895, following the ingestion of a large quantity of pears. The subsequent history was identical with the first attack. A slight induration in the right inguinal region was apparent, but disappeared shortly.

A third attack began on Dec. 30, 1895. This attack was much more marked than the previous attacks, the patient being sick for three weeks. A similar induration appeared by the fifth day but promptly subsided. The temperature in none of these attacks reached 100 , and the pulse at no time was above 90 . The pulse generally ran along between 72 and 80 . On Jan. 5,1896 , following an enema the patient passed a peculiar worm-like gelatinous "string" about twenty-four inches long, which was submitted to Dr. Walter Reed, U. S. A., who made the following report on the specimen:

$$
\begin{aligned}
& \text { War Department, Surgeon-General's Office. } \\
& \text { Washington, D. C., Jan. 8, } 1896 .
\end{aligned}
$$

Dear Doctor:--The specimen which you left at the Museum on Monday, the 6th inst., and which was said to have been passed from the bowel in a case of appendicitis, has been subjected to microscopic examination and found to consist of fine and coarse fibers of fibrin enclosing many necrotic and feebly staining columnar shaped cells. As far as we are able to judge microscopically I should say that the specimen consists of socalled membranous cast of the intestine.

$$
\text { Very sincerely yours, }
$$

Walter ReEd, Surgeon U. S. A., Curator.

No hemorrhage followed the passage of this specimen and the symptoms gradually disappeared. The patient was out in three weeks.

During an interval of nine months between the third and fourth attacks, the patient enjoyed excellent health and congratulated himself upon having entirely recovered. The fourth attack occurred suddenly on Sept. 14, 1896, while the patient was prepar- ing to leave the city on an extended tour. The symptoms being somewhat misleading and, but for the history of previous attacks, would not at first have attracted particular attention. A medical friend who was visiting the patient at that time, supposed the attack was one of simple indigestion, and administered a large dose of sulphate of magnesia. The temperature in this attack never rose above 99.4, pulse 72 . The patient was considered well in ten days and moved into a new house.

On the 25th the old symptoms showed themselves again and by the 26 th they were much more marked. On the 27th the patient had a chill which was not very pronounced, temperature reaching 101 and the pulse 80 . Pain in the side became acute and indura. tion with tension of the abdominal muscles in the right inguinal region, was well marked.

Operation was performed on the 28th, at which time the patient felt perfectly well, had no pain or soreness and none was produced by pressure over the inguinal region. The boggy sensation had, however extended, the temperature was 99 and the pulse 72, and the skin moist. The condition of the patient was decidedly misleading. The diagnosis of circumscribed appendical abscess, previously made was adhered to, and an incision resulted in the evacuation of about three quarts of greenish-black offensive pus. A small lump or mass was removed which was supposed to be the remains of the disorganized appendix, but was subsequently lost by the nurse, thus preventing an histologic examination. The abscess walls appeared to be gangrenous.

After an exploration, not endangering the exudate walls, the cavity was thoroughly irrigated with salt solution, and packed with iodoform gauze, which was permitted to remain forty-eight hours. Thorough aseptic precautions were observed in every detail. The patient made an uneventful convalescence, the wound healing promptly by granulation under gauze packing, giving a strong union without hernia.

I believe this case would have been lost through septic infection had an injudicious search been instituted further for an appendix which in all probability had sloughed away, and I re-affirm my opinion that all cases of appendicitis should be promptly operated upon.

On November 3 the patient passed an intestinal cast similar to the one above mentioned. This was submitted to Surgeon Walter Reed, who forwarded the following report.

War Department, Surgeon General's Office, Washington, D.C., Nov. 4, 1896.

Dear Doctor:-Referring to the specimen mentioned in your letter of Nov. 3, 1896, I beg to say that microscopic examina. tion shows it to consist of numerous interlacing fine fibers, enclosing in their meshes a large number of poorly staining cell-nuclei, and considerable granular amorphous material.

I think this specimen should be considered as a part of a membranous cast of the bowel. Sincerely yours,

Walter Reed, Surgeon U. S. A. Curator.

Baby's Turn Next Time.-Mrs. Briske-Johnny, did the doctor call while I was out?

Little Johnny (stopping his play)—Yes'm. He felt my pulse an' looked at my tongue, and shooked his head and said it was a very serious case, and he left this prescription and said he would call again before night.

Mrs. Briske-Gracious me! It wasn't you I sent him to see; it was the baby.-New York Weekly. 